Revista de Matemática: Teoría y Aplicaciones 2004 11(1) : 41-58

CIMPA - UCR - CCSS ISSN: 1409-2433

\title{
ALGORITMOS NUMÉRICOS PARA EL PROBLEMA DE RESTAURACIÓN DE IMÁGENES USANDO EL MÉTODO DE LAS PROYECCIONES ALTERNANTES
}

\author{
René Escalante*
}

Recibido/Received: 27 May 2003

\begin{abstract}
Resumen
Los algoritmos de proyección han evolucionado a partir del algoritmo de proyección alternante propuesto por J. von Neumann en 1933, donde el espacio solución es la intersección de un número finito de subespacios o conjuntos convexos. Investigaciones recientes se han centrado en técnicas para acelerar la convergencia del método y explotar el multiprocesamiento.

En este trabajo consideramos el problema de restauración de imágenes. La mayoría de las técnicas desarrolladas para resolverlo han usado algoritmos iterativos; una de ellas consiste en usar proyecciones ortogonales alternantes.

Llevamos a cabo una revisión cronológica de las diferentes técnicas en las que se ha aplicado el método de las proyecciones ortogonales alternantes al problema de restauración de imágenes, hasta llegar al enfoque reciente de Combettes (1997-1999), en donde la restauración se basa en el cálculo de proyecciones aproximadas, en lugar de proyecciones exactas.
\end{abstract}

Palabras clave: El problem de restauración de imágenes, el método de las proyecciones alternas, proyecciones ortogonales alternantes, proyecciones sobre convexos, proyección por subgradientes en paralelo.

\begin{abstract}
The projection algorithms have evolved from the alternating projection method proposed by J. von Neumann in 1933, who treated the problem of finding the projection of a given point in a Hilbert space onto the intersection of two closed subspaces. Recent researches have been centered in techniques for accelerate the convergence of
\end{abstract}

* Centro de Cálculo Científico y Tecnológico, Escuela de Computación, Facultad de Ciencias, Universidad Central de Venezuela, Apartado 47887, Los Chaguaramos, Caracas 1041-A, Venezuela. E-Mail: rescalan@kuaimare.ciens.ucv.ve 
the method and to exploit the multiprocessing.

In this work we considered the image restoration problem. In most techniques developed to solved it have used iterative algorithms; one of them consists of using alternating orthogonal projections.

We carried out one chronological looking back of different techniques in which has been applied the method of the alternating orthogonal projections to the problem of imagen restoration, until arriving at the recent approach of Combettes (1997-1999), on where the restoration process is based on the computation of approximate projections (i.e., subgradient projections), instead of exact projections.

Keywords: Image restoration problem, alternating projection method, alternating orthogonal projections, convex projections, parallel subgradient projection.

Mathematics Subject Classification: 46N40.

\section{Introducción}

En el proceso de restauración de imágenes el objetivo consiste en estimar la forma original de una imagen degradada, de manera tal que la restauración de la imagen sea un proceso que busque recobrar la imagen a partir de algún conocimiento, que a priori se tenga, del fenómeno de degradación [22]. Así, las técnicas de degradación tratan de modelar la degradación y aplicar un proceso inverso para recuperar la imagen original.

Las técnicas tradicionales de solución empleadas de manera extensiva en la recuperación de imágenes (ver, por ejemplo, [2] y [29]), proporcionan poca flexibilidad en cuanto a la incorporación de restricciones al planteamiento del problema. En este sentido, la noción de factibilidad constituye una alternativa relevante [12], [9].

Estudiamos aquí el denominado proceso de proyecciones ortogonales alternantes aplicado al problema de restauración de imágenes. Llevamos a cabo una cronología de algunas estimaciones teóricas y algoritmos que resuelven este problema. También consideramos el nuevo enfoque de Combettes denominado método de extrapolación de proyecciones por subgradientes en paralelo (extrapolated method of parallel subgradient projections), el cual introduce el concepto de las proyecciones aproximadas (approximate projections) [9], [10]. En este sentido, pretende éste ser un trabajo de recopilación de métodos (o estrategias), no tradicionales, y de data reciente, en el estudio numérico del problema de recuperación de imágenes (y, en particular, del problema de restauración de imágenes).

En el $\S 2$ describimos el proceso de restauración de imágenes usando el denominado método de las proyecciones ortogonales alternantes (MAP), propuesto por Youla [42] y Youla \& Web [43]. En el parágrafo 3 hacemos una revisión cronológica de varias extensiones del MAP, siempre tratadas como herramientas en la resolución aproximada del problema de recuperación de imágenes. En el $\S 4$ exponemos el nuevo enfoque debido a Patrick L. Combettes [9] para abordar el problema de restauración de imágenes. Por último, en el parágrafo 5 exponemos algunos comentarios finales. 


\section{Restauración de imágenes y el MAP}

En esta sección mostramos el primer intento (del que tengamos conocimiento) que busca aplicar el método de las proyecciones ortogonales alternantes (method of the alternating projections, MAP) al problema de restauración de imágenes. Dicha aplicación fue desarrollada originalmente en 1978 por Youla [42] para subespacios cerrados (en un espacio de Hilbert), y posteriormente, en 1982, fue extendido al caso convexo por Youla y Webb [43].

\subsection{Método de proyecciones ortogonales alternantes}

Este método en su versión original fue desarrollado por J. von Neumann en 1933 [40], desde entonces muchas y diversas aplicaciones del mismo han sido desarrolladas (ver, por ejemplo, [8], [11], [15], [17], [18]).

Sea $H$ un espacio de Hilbert y $M$ un subespacio cerrado de $H$. La proyección ortogonal en $M$ será denotada por $P_{M}$. En particular, $P_{M}$ es lineal, autoadjunta e idempotente. $P_{M}(x)$ es el punto en $M$ más cercano a $x$, es decir

$$
\left\|x-P_{M}(x)\right\|=d(x, M),
$$

donde

$$
d(x, M)=\inf \{\|x-y\|: y \in M\},
$$

y su propiedad característica es:

$$
\left\langle x-P_{M}(x), y\right\rangle=0, \quad \forall y \in M .
$$

El complemento ortogonal de $M$, denotado por $M^{\perp}$, es

$$
M^{\perp}=\{y \in H:\langle x, y\rangle=0, \text { si } x \in M\} .
$$

Se sabe que si $P_{A}$ y $P_{B}$ conmutan, entonces, la composición de operadores de proyección verifica que $P_{A} P_{B}=P_{B} P_{A}=P_{A \cap B}$. Von Neumann estaba interesado en saber lo que se podía decir del caso en que estos operadores no conmutaran, demostrando el siguiente resultado.

Teorema 2.1 (von Neumann, 1933 [40]) Si $P_{A}$ y $P_{B}$ no conmutan, entonces para cada $x \in H$

$$
\lim _{n \rightarrow \infty}\left(P_{B} P_{A}\right)^{n} x=P_{A \cap B} x .
$$

Este resultado sugiere un algoritmo, el conocido Método de las Proyecciones Alternantes (o MAP).

$$
\begin{array}{ll}
\multicolumn{2}{c}{x_{0}=x} \\
x_{n}=P_{B} P_{A} x_{n-1} & \left(\equiv\left(P_{B} P_{A}\right)^{n} x\right) \\
n=1,2, \ldots \ldots . & x_{n} \rightarrow P_{A \cap B} x
\end{array}
$$

La interpretación geométrica de este método consiste en que para encontrar la mejor aproximación al punto $x$ desde $A \cap B$, primero se proyecta $x$ sobre $A$, el elemento resultante es 
entonces proyectado sobre $B$, y se continúan proyectando los resultados alternadamente sobre $A$ y $B$. La utilidad práctica de este procedimiento está en que, en general, resulta mucho mas fácil cálcular las proyecciones individuales, sobre $A$ ó $B$, que calcular su proyección sobre $A \cap B$ [15], [16]. El resultado obtenido por von Neumann fue extendido posteriormente por Halperin, en [25], a una familia finita de subespacios cerrados (ver también [16]).

Los operadores de proyección tienen la propiedad de ser no expansivos, es decir, la distancia entre dos puntos proyectados nunca excede la distancia entre los dos puntos originales.

\subsection{El MAP aplicado al problema de restauración de imágenes}

Se persigue aquí establecer un esquema iterativo para resolver problemas de restauración de imágenes usando el método de las proyecciones ortogonales alternantes.

Para ello consideraremos dos subespacios cerrados $A$ y $B$ que están contenidos en un espacio de Hilbert $H$, y supondremos que un elemento $x \in H$, pertenece también al subespacio B, del cual sólo se conoce su proyección sobre el subespacio cerrado A, es decir $y=P_{A} x$. ¿Cómo reconstruiremos pues la imagen $x$ a partir de $y$ ? Por medio de un proceso inverso, se desea encontrar la mejor aproximación a la imagen original.

Es un hecho conocido que todo elemento $x$ en un espacio de Hilbert $H$ se puede escribir como $x=z+w$, donde $z \in A$ y $w \in A^{\perp}$ (subespacio ortogonal de $A$ ), lo que nos lleva a observar que la proyección sobre el subespacio cerrado $A$ se puede escribir como $P_{A}=\left(I-P_{A^{\perp}}\right)$.

Además, es claro que si $x \in B$, su proyección sobre el subespacio cerrado $B$ es él mismo; es decir, $x=P_{B} x$.

Así, con el objeto de tratar de restaurar la imagen (por puntos) $x$ a partir de su proyección sobre el subespacio cerrado $A$, vemos primero que

$$
\begin{aligned}
y & =P_{A} x \\
& =P_{A} P_{B} x \\
& =\left(I-P_{A^{\perp}}\right) P_{B} x \\
& =P_{B} x-P_{A^{\perp}} P_{B} x \\
& =x-P_{A^{\perp}} P_{B} x .
\end{aligned}
$$

De manera que, para que exista una solución del problema planteado, $y$ debe estar en el rango de $\left(I-P_{A^{\perp}} P_{B}\right)$. Mas aún, $x=y+P_{A^{\perp}} P_{B} x$. Lo cual sugiere el siguiente esquema iterativo:

$$
\begin{gathered}
x_{1}=y \\
x_{n+1}=y+P_{A^{\perp}} P_{B} x_{n} \quad n=1,2, \ldots .
\end{gathered}
$$

Podemos obtener un esquema iterativo equivalente haciendo uso de algunos hechos ya verificados:

$$
\begin{aligned}
& \text { 1.- } x_{n+1}=y+P_{A^{\perp}} P_{B} x_{n} \\
& \text { 2.- } y=x-P_{A^{\perp}} P_{B} x
\end{aligned}
$$


3.- $\lim _{n \rightarrow \infty}\left(P_{A} P_{B}\right)^{n} x=P_{A \cap B} x$

4.- $x_{1}=y$

Aplicando 1.- de manera sucesiva, y suponiendo 2.-, obtenemos

$$
x_{n+1}=x-\left(P_{A^{\perp}} P_{B}\right)^{n} x+\left(P_{A^{\perp}} P_{B}\right)^{n} x_{1}
$$

Y por 3.- y 4.-, llegamos a que

$$
\lim _{n \rightarrow \infty} x_{n+1}=x-P_{A^{\perp} \cap B} x+P_{A^{\perp} \cap B} y
$$

Este esquema iterativo converge a $x$, que es la solución del problema, si $A^{\perp} \cap B=\{0\}$ ó equivalentemente si $\left\|P_{A^{\perp}} P_{B}\right\|<1$. Si ninguna de estas dos condiciones se cumple no podemos asegurar la convergencia del esquema iterativo.

Si $A^{\perp} \cap B=\{0\}$, entonces la proyección sobre esta intersección es el elemento cero, por lo que $P_{A^{\perp} \cap B} x$ y $P_{A^{\perp} \cap B} y$ serían iguales a cero, lo que implicaría que el esquema iterativo converge a $x$.

Ahora, si $\left\|P_{A^{\perp}} P_{B}\right\|<1$, entonces sigue de (1) que

$$
x_{n+1}-x=\left(P_{A^{\perp}} P_{B}\right)^{n} y-\left(P_{A^{\perp}} P_{B}\right)^{n} x .
$$

Aplicando norma a ambos lados queda

$$
\left\|x_{n+1}-x\right\|=\left\|\left(P_{A^{\perp}} P_{B}\right)^{n} y-\left(P_{A^{\perp}} P_{B}\right)^{n} x\right\|,
$$

de donde

$$
\left\|x_{n+1}-x\right\|=\left\|\left[\left(P_{A^{\perp}} P_{B}\right)^{n}\right](y-x)\right\|,
$$

y por propiedades de la norma:

$$
\begin{aligned}
\left\|x_{n+1}-x\right\| & \leq\left\|\left(P_{A^{\perp}} P_{B}\right)^{n}\right\|\|y-x\| \\
& =\left\|\left(P_{A^{\perp}} P_{B}\right)\left(P_{A^{\perp}} P_{B}\right) \ldots\left(P_{A^{\perp}} P_{B}\right)\right\|\|y-x\| \\
& \leq\left\|P_{A^{\perp}} P_{B}\right\|\left\|P_{A^{\perp}} P_{B}\right\| \ldots\left\|P_{A^{\perp}} P_{B}\right\|\|y-x\| \\
& =\left\|P_{A^{\perp}} P_{B}\right\|^{n}\|y-x\|
\end{aligned}
$$

Como $\left\|P_{A^{\perp}} P_{B}\right\|<1$, al hacer $n \rightarrow \infty$ la expresión tiende a cero, por lo que el lado derecho de la desigualdad tiende a cero y $\left\|x_{n+1}-x\right\| \rightarrow 0$, cuando $n \rightarrow \infty$.

Para detalles adicionales, teoremas de convergencia y algunos ejemplos, ver [42]

\subsection{Restauración de imágenes y el MAP en el caso convexo}

En el estudio del problema de restauración de imágenes por el método de las proyecciones alternantes para el caso de subespacios cerrados, nos basamos en el hecho de que los operadores de proyección son lineales.

Supondremos ahora que cada restricción conocida de la imagen original (nos referimos a cada propiedad de la imagen) está definida por un conjunto cerrado y convexo, y que la intersección de los conjuntos así definidos también es un conjunto convexo y cerrado. 
En el caso de proyecciones sobre subespacios cerrados no se admiten restricciones (propiedades de la imagen) no lineales, y debido a que en la mayoría de los casos los algoritmos de restauración de imágenes trabajan con información incompleta, se planteará, para el caso convexo, un algoritmo que, en esencia, también es iterativo (recursivo) y admite cualquier número de restricciones no lineales de un cierto tipo.

La base conceptual del algoritmo de restauración de imágenes por el método de las proyecciones ortogonales alternantes sobre conjuntos convexos, en un espacio de Hilbert $H$, es similar al caso de subespacios cerrados en un espacio de Hilbert $H$. Suponemos pues que el elemento original $x$ pertenece a $S_{0}$, que no es más que la intersección de $m$ conjuntos convexos cerrados y bien definidos, digamos $S_{1}, S_{2}, \ldots ., S_{m}$; o bien,

$$
x \in S_{0}=\cap_{i=1}^{m} S_{i}
$$

De manera que, contando solamente con la información de los correspondientes operadores de proyección $P_{i}$ (no lineales) sobre cada uno de los conjuntos convexos $S_{i}(i=1,2, \ldots, m)$, se desea restaurar la imagen $x$, preferiblemente usando algún esquema iterativo.

Es importante observar que, por lo general, $S_{0}$ puede ser muy complejo en estructura, por lo que tener un operador de proyección $P_{0}$ sobre $S_{0}$ usualmente no es posible. De manera que, debemos buscar resolver nuestro problema a partir de las proyecciones $P_{i}$, $i=1, \ldots, m$.

Cuando tratamos con proyecciones sobre conjuntos convexos debemos tener presente el siguiente resultado, el cual no es más que una extensión del conocido teorema de proyecciones ortogonales para subespacios cerrados (ver, por ejemplo, [30] o [16]).

Teorema 2.2 Sea $x$ un vector en un espacio de Hilbert $H$ y sea $C$ un subconjunto convexo cerrado de $H$. Entonces, existe un único $c \in C$ tal que

$$
\|x-c\|=\inf _{z \in C}\|x-z\| .
$$

Más aún, una condición necesaria y suficiente para que c sea el único vector minimizador está dada por el denominado criterio de Kolmogorov:

$$
\langle x-c, z-c\rangle \leq 0, \quad \forall z \in C
$$

Denotaremos a este vector por $c=P_{C} x$, y diremos que $P_{C}$ es el operador asociado que proyecta $H$ sobre $C$. Si $x \in C$, entonces $P_{C} x=x$, así que todo elemento de $C$ es un punto fijo para el operador de proyección $P_{C}$ sobre ese conjunto.

Consideremos el siguiente operador composición:

$$
T=P_{m} P_{m-1} \ldots P_{2} P_{1}
$$

Aunque, en general, $T$ no es un operador de proyección sobre $S_{0}$, todo punto de $S_{0}$ es un punto fijo para cada $P_{i}$ y, por lo tanto, de $T$ (i.e., si $x \in S_{0}$, entonces $x \in S_{i}$, $\forall i=1,2, \ldots, m$, por lo que $P_{i} x=x, \forall i=1,2, \ldots, m$, de donde $\left.T x=x\right)$. Recíprocamente, si $S_{0} \neq \emptyset$, todo punto fijo de T es un punto que está en $S_{0}$. 
De lo anterior, resulta claro que un esquema iterativo sencillo para generar puntos fijos de T podría ser el siguiente:

$$
\begin{gathered}
x_{n}=T^{n} x \\
n=0,1,2, \ldots, \quad x \text { arbitrario }
\end{gathered}
$$

En efecto, $T^{n} x$ converge a $x$, pero no a una velocidad geométrica [43]. Para mayores detalles acerca de la convergencia del esquema propuesto sugerimos al lector revisar el trabajo de Youla y Webb [43], y para conocer más acerca de algunas aplicaciones y de la experimentación numérica relacionada ver [36].

\section{Métodos de proyección con extrapolación}

Para resolver el problema teórico de recuperación ${ }^{1}$ de imágenes sobre conjuntos convexos en un espacio de Hilbert bastará con encontrar un punto en la intersección de los mismos (i.e., el problema de factibilidad convexa). Una importante herramienta para resolver estos problemas es el algoritmo de las proyecciones sucesivas sobre conjuntos convexos (i.e., the algorithm of the periodic projections onto convex sets ó POCS [12], [9]).

En este contexto el espacio solución $H$ es un espacio de Hilbert, en el cual la imagen es descrita únicamente por una familia de restricciones convexas $\left(\Psi_{i}\right)_{i \in I}$, de manera tal que la familia de conjuntos convexos y cerrados involucrados en el problema tiene la forma

$$
(\forall i \in I) \quad S_{i}=\left\{a \in H: a \text { satisface } \Psi_{i}\right\} .
$$

De esta forma, el problema de recuperación se transforma en el problema de factibilidad convexa

$$
\text { Encontrar } a^{*} \in S=\bigcap_{i \in I} S_{i}
$$

Un método sencillo para resolver este problema es el POCS. Si asumimos que la familia de conjuntos es finita, es decir $I=\{1, \ldots, m\}$, el método genera una imagen en $S$ como el límite de una sucesión $\left(a_{n}\right)_{n \geq 0}$ de proyecciones periódicas sobre los conjuntos involucrados; es decir,

$$
(\forall n \in \mathbb{N}) \quad a_{n+1}=P_{n(\bmod m)+1}\left(a_{n}\right)
$$

donde $P_{i} \equiv P_{S_{i}}$. La popularidad de este algoritmo se ve ensombrecida por los siguientes inconvenientes de tipo teórico y numéricos [9]:

- Converge lentamente.

- Sólo puede procesar un conjunto por iteración, por lo cual no es adecuado para implantación en procesadores paralelos.

- Requiere del cálculo de una proyección exacta en cada iteración.

\footnotetext{
${ }^{1}$ El problema de recuperación de imágenes plantea estimar una imagen a partir de señales, física o matemáticamente relacionadas con ella. En este sentido, se habla tanto de restauración como de reconstrucción de imágenes.
} 
- Está limitado a problemas con un número finito de restricciones.

Se desea introducir un método de proyecciones en paralelo que supere las dificultades arriba mencionadas. Este método iterativo, denominado método de extrapolación de proyecciones por subgradientes en paralelo (i.e., extrapolated method of parallel subgradient projections ó EMOPSP) fue propuesto por Combettes en [9], [10] (ver $\S 4$ ).

\subsection{Métodos de proyección}

Haremos aquí un breve recuento de diferentes versiones de los métodos de proyección que, en los últimos años, algunos investigadores han propuesto y aplicado al área de recuperación de imágenes. Como antes, asumiremos que $\left(S_{i}\right)_{i \in I}$ es una familia finita de $m$ conjuntos convexos y cerrados.

\subsubsection{Proyecciones periódicas sobre convexos (POCS)}

Al comienzo del $\S 3$ consideramos el esquema iterativo (3), el cual fue conocido inicialmente con el nombre de técnica de reconstrucción algebraica (algebraic reconstruction technique, ART) para encontrar una imagen en la intersección de un número finito de hiperplanos [23]. En su forma más general, el algoritmo POCS está descrito por el algoritmo

$$
(\forall n \in \mathbb{N}) \quad a_{n+1}=a_{n}+\lambda_{n}\left(P_{n(\bmod m)+1}\left(a_{n}\right)-a_{n}\right),
$$

donde los parámetros de relajación satisfacen:

$$
(\forall n \in \mathbb{N}) \quad \varepsilon \leq \lambda_{n} \leq 2-\varepsilon, \quad \text { con } 0<\varepsilon<1 .
$$

El principal inconveniente del método es su lenta convergencia, especialmente cuando el ángulo entre los conjuntos involucrados es pequeño (esto fue reportado en [24]). El parámetro $\lambda_{n}$ puede ser $\leq 1$ (forma sub-relajada) ó $\geq 1$ (forma sobre-relajada). Desafortunadamente, esta flexibilidad no puede ser explotada para acelerar las iteraciones, por lo que no hay una respuesta precisa sobre si la sub-relajación es más rápida que la sobre-relajación o viceversa [26], [31], [38].

\subsubsection{Técnica de reconstrucción iterativa simultánea}

La Técnica de Reconstrucción Iterativa Simultánea (Simultaneous Iterative Reconstruction Technique, SIRT) fue desarrollada en [21] para la reconstrucción de imágenes tomográficas. En este método, considerado como la versión paralela del ART, las proyecciones del iterado actual sobre todos los conjuntos (en este caso hiperplanos) son promediadas para obtener el nuevo iterado, esto es

$$
(\forall n \in \mathbb{N}) \quad a_{n+1}=\frac{1}{m} \sum_{i \in I} P_{i}\left(a_{n}\right)
$$

Aunque el SIRT dió mejores resultados que el ART en ambientes con ruido, se ha comprobado que su convergencia no es tan rápida [1], [26]. 


\subsubsection{El método de proyección en paralelo}

El Método de Proyección en Paralelo (Parallel Projection Method, PPM) es una generalización del SIRT y tiene la expresión recursiva:

$$
(\forall n \in \mathbb{N}) \quad a_{n+1}=a_{n}+\lambda_{n}\left(\sum_{i \in I} w_{i} P_{i}\left(a_{n}\right)-a_{n}\right),
$$

donde los parámetros de relajación satisfacen (4) y

$$
\sum_{i \in I} w_{i}=1 \quad \text { y } \quad(\forall i \in I) w_{i}>0
$$

El PPM fue considerado inicialmente para problemas de infactibilidad convexa $(S=\emptyset)$ [13], resolviendo el problema (2), para el caso $S \neq \emptyset$, con una convergencia más rápida que POCS cuando usamos sobre-relajación [13].

\subsubsection{El método de proyecciones paralelas}

Aunque el PPM es útil para problemas inconsistentes, no es muy flexible como método paralelo, ya que requiere que todos los conjuntos esten activos en cada iteración. Como consecuencia de ello, si el número de conjuntos es mayor que la cantidad de procesadores disponibles, la implantación no será óptima. Para mejorar la carga computacional en cada iteración entre los procesadores, es deseable tener la posibilidad de activar subfamilias variables de conjuntos. El Método de Proyecciones Paralelas (Method of Parallel Projections, MOPP) cumple con este requerimiento y está descrito por el algoritmo

$$
(\forall n \in \mathbb{N}) \quad a_{n+1}=a_{n}+\lambda_{n}\left(\sum_{i \in I_{n}} w_{i, n} P_{i}\left(a_{n}\right)-a_{n}\right),
$$

donde estamos considerando (4), y donde la sucesión de control $\left(I_{n}\right)_{n \geq 0}$ impone la condición de que todo conjunto sea activado al menos una vez durante cualquier ciclo de $M$ iteraciones consecutivas Según [9] el primer estudio de fórmulas recursivas del tipo (5), en espacios de Hilbert, fue dado en [33]. Asimismo, en [7] encontramos aplicaciones de imágenes médicas usando algoritmos en paralelo que procesan bloques de restricciones en las iteraciones.. El MOPP tiene como casos particulares los algoritmos presentados previamente ${ }^{2}$ (POCS y PPM).

Intuitivamente, un algoritmo de proyección paralelo es más eficiente numéricamente que un algoritmo secuencial, ya que las proyecciones pueden ser procesadas simultáneamente. Desafortunadamente, este no es siempre el caso para métodos no relajados tales como el SIRT, los cuales a menudo son más lentos que el POCS. Una ventaja de los métodos de proyecciones paralelas es que ellos pueden ser acelerados vía sobre-relajaciones [14], [13], $[28]$.

${ }^{2}$ El MOPP también generaliza al denominado algoritmo de Cimmino (ANCA) [28]. En [28] se estudió el ANCA para el caso de dimensión finita, mostrando ser más rápido que el SIRT. 


\subsection{El problema de factibilidad convexa en un espacio producto}

En Teoría de Optimización, el concepto de espacio producto ha sido usado para descomponer problemas de minimización con múltiples restricciones en una sucesión de problemas elementales con una sóla restricción. La reformulación del problema de factibilidad (2) como un problema que involucra dos conjuntos en $\mathbf{H} \equiv H^{m}, m<\infty$, (el producto cartesiano del espacio original $H$ ) fue tratado inicialmente en [34] (fue también usado en [13] para resolver problemas de factibilidad de señales inconsistentes). Esta formulación permite una estructura conveniente para desarrollar los denominados métodos de proyección extrapolados.

\subsubsection{Método de proyección en paralelo extrapolado}

Siguiendo a [9], [34], observamos primero que el problema de factibilidad (2) es equivalente a resolver el siguiente problema más sencillo (en el que solamente consideramos dos conjuntos)

$$
\text { Encontrar } \mathbf{a}^{*} \in \mathbf{S} \bigcap \mathbf{D}
$$

donde

$$
\mathbf{S}=\times_{i \in I} S_{i}=\left\{\mathbf{a} \in \mathbf{H}:(\forall i \in I) a_{i} \in S_{i}\right\} \quad \text { y } \quad \mathbf{D}=\{(a, \ldots, a) \in \mathbf{H}: a \in H\} .
$$

Para hallar $a^{*}$, fijamos $a_{0} \in \mathbf{D}$ y construímos una sucesión $\left(a_{n}\right)_{n \geq 0} \subset \mathbf{D}$ vía el esquema de proyecciones alternantes, llamado Método de Proyección en Paralelo Extrapolado (Extrapolated Parallel Projection Method, EPPM):

$$
(\forall n \in \mathbb{N}) \quad \mathbf{a}_{n+1}=\mathbf{a}_{n}+\lambda_{n}\left(P_{\mathbf{D}} \circ P_{\mathbf{S}}\left(\mathbf{a}_{n}\right)-\mathbf{a}_{n}\right)
$$

Como este algoritmo es un caso especial del POCS, obtenemos inmediatamente el siguiente resultado.

Teorema 3.1 Toda sucesión $\left(\mathbf{a}_{n}\right)_{n \geq 0}$ construída aplicando (6), con estrategia de relajación (4) converge débilmente a un punto en $\mathbf{S} \cap \mathbf{D}$.

A partir del teorema anterior y de las definiciones dadas no es difícil llegar al siguiente teorema.

Teorema 3.2 (Pierra, 1984 [34]) Toda sucesión $\left(\mathbf{a}_{n}\right)_{n \geq 0}$ generada por (6) con estrategia de relajación

$$
(\forall n \in \mathbb{N}) \quad \varepsilon \leq \lambda_{n} \leq L_{n}, \quad \text { con } \quad 0<\varepsilon<1,
$$

donde

$$
L_{n}=\frac{\sum_{i \in I} w_{i}\left\|P_{i}\left(a_{n}\right)-a_{n}\right\|^{2}}{\left\|\sum_{i \in I} w_{i} P_{i}\left(a_{n}\right)-a_{n}\right\|^{2}}
$$

converge débilmente a un punto en $\mathbf{S} \cap \mathbf{D}$. 
Demostración: Para los detalles sugerimos al lector revisar las referencias [9] ó [34].

Consecuencia de estos resultados, son los dos teoremas siguientes (ver [34] para detalles):

Teorema 3.3 Todo ciclo $\left(a_{n}\right)_{n \geq 0}$ del método PPM converge débilmente a un punto en $\mathbf{S}$.

Teorema 3.4 Todo ciclo $\left(a_{n}\right)_{n \geq 0}$ del método EPPM converge débilmente a un punto en S.

Se observó en [34] que la rápida convergencia del algoritmo EPPM se debió a las "mayores sobre-relajaciones" permitidas por (7), ya que el parámetro de relajación puede alcanzar valores mayores que 2 , atenuando, de esta forma, el "problema del ángulo".

\subsubsection{Generalización del método de proyección en paralelo extrapolado}

Hasta ahora hemos podido observar que el método EPPM y los anteriores requieren del cálculo de $m$ proyecciones exactas. Como los subproblemas de optimización involucrados son a menudo díficiles de resolver, Combettes [9] propuso el uso de las denominadas proyecciones aproximadas (ver Lema 4.1 y observaciones siguientes).

En principio, reemplazamos las proyecciones exactas de $\mathbf{a}_{n}$ en $\mathbf{S}$ por una proyección aproximada $P_{\mathbf{S}_{n}}\left(\mathbf{a}_{n}\right)$, de manera que

$$
(\forall n \in \mathbb{N}) \quad \mathbf{a}_{n+1}=\mathbf{a}_{n}+\lambda_{n}\left(P_{\mathbf{D}} \circ P_{\mathbf{S}_{n}}\left(\mathbf{a}_{n}\right)-\mathbf{a}_{n}\right),
$$

donde $\mathbf{a}_{0} \in \mathbf{D}$ y $\left(\mathbf{S}_{n}\right)_{n \geq 0}$ es una sucesión de conjuntos convexos y cerrados de $\mathbf{H}$ tales que

$$
(\forall n \in \mathbb{N}) \quad \mathbf{S} \subset \mathbf{S}_{n} \quad \text { y } \quad \mathbf{a}_{n} \notin \mathbf{S}_{n}-\mathbf{S} .
$$

En otras palabras, $\mathbf{S}_{n}$ es un "superconjunto" de $\mathbf{S}$, el cual contiene a $\mathbf{a}_{n}$ sólo cuando $\mathbf{S}$ lo hace.

La estrategia de relajación será como sigue ${ }^{3}$ :

$$
(\forall n \in \mathbb{N}) \quad \varepsilon \leq \lambda_{n} \leq(2-\varepsilon) L_{n}, \quad \text { con } \quad 0<\varepsilon<1,
$$

donde

$$
L_{n}=\frac{\sum_{i \in I} w_{i}\left\|P_{i, n}\left(a_{n}\right)-a_{n}\right\|^{2}}{\left\|\sum_{i \in I} w_{i} P_{i, n}\left(a_{n}\right)-a_{n}\right\|^{2}}
$$

y donde, para todo $i \in I,\left(P_{i, n}\right)_{n \geq 0}$ es una sucesión de operadores de proyección sobre conjuntos convexos cerrados $\left(S_{i, n}\right)_{n \geq 0}$ tales que

$$
(\forall n \in \mathbb{N}) \quad S_{i} \subset S_{i, n} \quad \text { y } \quad a_{n} \notin S_{i, n}-S_{i} .
$$

\footnotetext{
${ }^{3}$ Note el lector que también la extensión del EPPM ha incluído aquí el uso de una relajación hasta $2 L_{n}$. En consecuencia, esperamos obtener una convergencia más rápida en determinados problemas, debido al uso de mayores relajaciones que las permitidas por PPM y EPPM.
} 
El esquema (8) en $\mathbf{H}$ produce un método de proyecciones en paralelo en $H$, que en [9] denominan EPPM2, y está definido por

$$
(\forall n \in \mathbb{N}) \quad a_{n+1}=a_{n}+\lambda_{n}\left(\sum_{i \in I} w_{i} P_{i, n}\left(a_{n}\right)-a_{n}\right),
$$

donde asumimos (9).

La ventaja del EPPM2 sobre el MOPP está en su capacidad de usar proyecciones aproximadas y en poder utilizar relajaciones más grandes. Esto significa que el EPPM2 converge en menos iteraciones y que el costo computacional de cada iteración es menor. Por otra parte, el MOPP es más flexible que el EPPM2 en el sentido de que puede procesar un número variable de conjuntos por iteración.

\section{El enfoque de Combettes}

Revisaremos a continuación el método iterativo, denominado método de extrapolación de proyecciones por subgradientes en paralelo (i.e., extrapolated method of parallel subgradient projections, EMOPSP), el cual fue propuesto por Combettes en [9]. Nos referimos al EMOPSP al comienzo del $\S 3$, cuando observamos una serie de inconvenientes (de corte teórico y numéricos) que presenta el método clásico (3).

Este método combina las ventajas de los métodos MOPP y EPPM2 con el uso de las proyecciones por subgradientes, lo cual permite una forma sencilla de calcular explícitamente las proyecciones aproximadas.

\subsection{El método de extrapolación de proyecciones por subgradientes en paralelo}

El Método de Extrapolación de Proyecciones por Subgradientes en Paralelo (Extrapolated Method of Parallel Subgradient Projections, EMOPSP) genera una sucesión $\left(a_{n}\right)_{n \geq 0}$ de imágenes de la siguiente manera: En la iteración $n$ se calculan y se promedian las proyecciones aproximadas $\left(P_{i, n}\left(a_{n}\right)\right)_{i \in I_{n}}$ del iterado actual $a_{n}$ sobre una subfamilia de conjuntos $\left(S_{i}\right)_{i \in I_{n} \subset I}$, vía una combinación convexa para formar $d_{n}=\sum_{i \in I_{n}} w_{i, n} P_{i, n}\left(a_{n}\right)$. Las proyecciones aproximadas son estimadas como proyecciones por subgradientes, de manera que todas las operaciones de proyección serán lineales, determinándose además un parámetro de extrapolación $L_{n} \geq 1$, de donde la actualización se obtiene por $a_{n+1}=a_{n}+\lambda_{n}\left(d_{n}-a_{n}\right)$, y $\lambda_{n} \in\left[0,2 L_{n}\right]$.

\subsubsection{Proyecciones por subgradientes}

Revisaremos primero algunas definiciones del área de análisis convexo. Sea $A$ un subconjunto cerrado y convexo, no vacío, de $H$, y sea $a$ un vector en $H$. Consideremos un semi-espacio afín $U=\{h \in H:\langle h, b\rangle \leq \eta, b \neq 0 \quad$ y $\eta \in \mathbb{R}\}$ tal que $a \notin U \supset A$. Entonces el hiperplano afín $V=\{h \in H:\langle h, b\rangle=\eta\}$ (asociado al semiespacio $U$ ) separa $a$ y $A, \mathrm{y}$

$$
P_{U}(a)=P_{V}(a)=a+\frac{\eta-\langle a, b\rangle}{\|b\|^{2}} b .
$$


Notemos que si $P_{A}(a) \in V$, entonces $V$ soporta a $A$ en $P_{A}(a)$.

Definición 4.1 Sea $g: S \rightarrow \mathbb{R}$ una función convexa. La $\mu$-sección $(\mu \in \mathbb{R})$, la gráfica $y$ el epígrafe de $g$ están definidos, respectivamente, por

$$
\begin{aligned}
& \sec (g, \mu)=\{x \in H: g(x) \leq \mu\}, \\
& \operatorname{gr} g=\{(x, \mu) \in H \times \mathbb{R}: g(x)=\mu\},
\end{aligned}
$$$$
\text { epi } g \equiv\{(x, \mu) \in H \times \mathbb{R}: g(x) \leq \mu\} \text {, }
$$

Definición 4.2 Sea $S$ un conjunto convexo no vacío de $H$. Decimos que un vector $\xi$ es un subgradiente ([32]) de $g$ en $a \in S$ si

$$
(\forall b \in S) \quad g(b) \geq\langle b-a, \xi\rangle+g(a)
$$

El vector subgradiente corresponde a la "pendiente" del hiperplano de soporte de epi $g$ en $(a, g(a)) \in H \times \mathbb{R}$, definido por la función $g(a)+\langle b-a, \xi\rangle$.

Definición 4.3 El subdiferencial de g en a es el conjunto de sus subgradientes. Es decir,

$$
\partial g(a)=\{\xi \in H:(\forall b \in H)\langle b-a, \xi\rangle \leq g(b)-g(a)\}
$$

Si $g$ es continua en $a, g$ es subdiferenciable en $a(\partial g(a) \neq \emptyset)$. Si $g$ es diferenciable (Gâteaux) en $a$, entonces existe un único subgradiente, $\nabla g(a)$ (el gradiente). Si $g=\mathrm{d}(., A)$, sigue que, para todo $a$ en el complemento de un conjunto $A$,

$$
\nabla \mathrm{d}(a, A)=\frac{a-P_{A}(a)}{\left\|a-P_{A}(a)\right\|}
$$

Para revisar conceptos y resultados adicionales del campo del análisis convexo, el lector puede consultar, por ejemplo, las referencias [4], [6] y [35].

Observemos a continuación que cada restricción convexa puede usualmente expresarse a través de una desigualdad convexa, y que al conjunto $S_{i}$ asociado lo podemos escribir como una 0-sección

$$
S_{i}=\sec \left(g_{i}, 0\right)=\left\{a \in H: g_{i}(a) \leq 0\right\}
$$

de una funcional convexa semi-continua $g_{i}: H \rightarrow \mathbb{R}$. En algunos casos, la proyección $P_{i}\left(a_{n}\right)$ de una imagen $a_{n}$ en el complemento de $S_{i}$ puede resolverse relativamente fácil y admite una solución en forma cerrada (ver, por ejemplo, [43] ó [17]). En otros casos, sin embargo, la proyección exacta no es conocida (ver, por ejemplo, [37] ó [39]). El candidato natural para el conjunto $S_{i, n}$ es el semi-espacio afín que contiene a $S_{i}$ pero no a $a_{n}$. Así, $P_{i, n}\left(a_{n}\right)$ es simplemente la proyección sobre el hiperplano $V_{i, n}$ (asociado a $S_{i, n}$ ) que separa $a_{n}$ y $S_{i}$. El hecho práctico clave en este enfoque será la determinación eficiente del hiperplano de separación $V_{i, n}$.

Lema 4.1 (Combette, [9]) Sea $\xi_{i, n} \in \partial g_{i}\left(a_{n}\right)$. Entonces, el semi-espacio $S_{i, n}=\{a \in$ $\left.H:\left\langle a_{n}-a, \xi_{i, n}\right\rangle \geq g_{i}\left(a_{n}\right)\right\}$ es una aproximación de $S_{i}$ en el sentido de (10). 
Demostración: De (13) sigue que, si $a_{n} \notin S_{i}$, entonces $g_{i}\left(a_{n}\right)>0$ y $a_{n} \notin S_{i, n}$ (pues de lo contrario, $\left.\left\langle a_{n}-a_{n}, \xi_{i, n}\right\rangle \geq g_{i}\left(a_{n}\right)>0\right)$. Además, si $a \in S_{i}$, entonces $g_{i}(a) \leq 0 \mathrm{y}$, por lo tanto, $\left\langle a_{n}-a, \xi_{i, n}\right\rangle \geq g_{i}\left(a_{n}\right)-g_{i}(a) \geq g_{i}\left(a_{n}\right)$, por lo que $a \in S_{i, n}$. De donde sigue que $S_{i} \subset S_{i, n}$.

\section{Observaciones:}

- De (11) sigue que la proyección de $a_{n}$ (en el complemento de $S_{i}$ ) sobre $S_{i, n}$ está dada por

$$
P_{i, n}\left(a_{n}\right)=a_{n}-\frac{g_{i}\left(a_{n}\right)}{\left\|\xi_{i, n}\right\|^{2}} \xi_{i, n}
$$

la cual denominamos una proyección por subgradiente. De manera que, sólo requerimos del cálculo del subgradiente $\xi_{i, n}$ para realizar la proyección aproximada (en lugar de calcular la proyección exacta $P_{i}\left(a_{n}\right)$ ).

- En la práctica, $g_{i}$ será, por lo general, diferenciable, por lo que $\xi_{i, n}=\nabla g_{i}\left(a_{n}\right)$.

- Si contamos con $P_{i}\left(a_{n}\right)$, podemos hacer $g_{i}\left(a_{n}\right)=d\left(a_{n}, S_{i}\right)$, y (14), junto con (12), da la proyección exacta.

\subsubsection{Algoritmo y convergencia}

\section{Algoritmo EMOPSP [9]:}

Entrada: $a_{0} \in H, M \in \mathbb{N}^{*}, 0<\delta<1 / M, 0<\varepsilon<1$.

Hacer

$$
(\forall n \in \mathbb{N}) \quad a_{n+1}=a_{n}+\lambda_{n}\left(\sum_{i \in I_{n}} w_{i, n} P_{i, n}\left(a_{n}\right)-a_{n}\right),
$$

donde, para cada (iteración) $n \in \mathbb{N}$,

i) La familia de índices $I_{n}$ satisface $^{4}$

$$
I_{n} \subset I, I_{n} \neq \emptyset \text { y } \operatorname{card}\left\{i \in I_{n}: a_{n} \notin S_{i}\right\} \leq M .
$$

ii) Las proyecciones por subgradientes $\left(P_{i, n}\left(a_{n}\right)\right)_{i \in I_{n}}$ están definidas por

$$
P_{i, n}\left(a_{n}\right)= \begin{cases}a_{n}-\frac{g_{i}\left(a_{n}\right)}{\left\|\xi_{i, n}\right\|^{2}} \xi_{i, n} & \text { si } a_{n} \notin S_{i}, \\ a_{n} & \text { en caso contrario. }\end{cases}
$$

iii) Los pesos $\left(w_{i, n}\right)_{i \in I_{n}}$ satisfacen $^{5}$

$$
\left\{\begin{array}{l}
\sum_{i \in I_{n}} w_{i, n}=1 \\
\left(\forall i \in I_{n}\right)
\end{array} w_{i, n} \geq \delta \chi_{\text {complemento } S_{i}}\left(a_{n}\right) .\right.
$$

iv) $\varepsilon \leq \lambda_{n} \leq(2-\varepsilon) L_{n}$, donde

$$
L_{n}= \begin{cases}\frac{\sum_{i \in I_{n}} w_{i, n}\left\|P_{i, n}\left(a_{n}\right)-a_{n}\right\|^{2}}{\left\|\sum_{i \in I_{n}} w_{i, n} P_{i, n}\left(a_{n}\right)-a_{n}\right\|^{2}} & \text { si } a_{n} \notin \cap_{i \in I_{n}} S_{i}, \\ 1 & \text { en caso contrario }\end{cases}
$$

\footnotetext{
${ }^{4}$ La cardinalidad de un conjunto $A$ será denotada por $\operatorname{card} A$.

${ }^{5}$ Aquí la función característica de un conjunto $A$ será denotada por $\chi_{A}$.
} 
$\underline{\text { Salida: }}$ Se genera una sucesión de iterados $\left(a_{n}\right)_{n \geq 0}$, hasta que se cumpla algún criterio de $\operatorname{parada}^{6}$.

Los términos en que se plantea el siguiente resultado son los requeridos por las aplicaciones en el área de restauración de imágenes (i.e., un número de restricciones finito e imágenes discretizadas sobre un dominio acotado).

Teorema 4.1 (Combettes, 1997 [9]) Supongamos que dim $H<\infty$ y cardI $<+\infty$. Entonces, para cualquier familia de funcionales convexos $\left(g_{i}\right)_{i \in I}$, cualquier sucesión generada por el Algoritmo EMOPSP converge a una imagen factible, con tal que todos los conjuntos sean usados de manera repetida y en cualquier orden.

\section{Comentarios finales}

En este trabajo revisamos cómo un grupo de métodos de proyecciones ortogonales alternas son aplicados al problema de recuperación de imágenes (en particular, al problema de restauración de imágenes).

Comenzamos este artículo considerando los trabajos clásicos de Youla [42] y Youla y Webb [43]. Revisamos después, y de una manera cronológica, una serie de conocidas extensiones del MAP, en el contexto del problema de recuperación de imágenes. Por último, finalizamos la exposición con el aporte de Combettes [9]: el método de proyección EMOPSP, que resuelve el problema de factibilidad convexa asociado al problema de recuperación de imágenes, a través de la aplicación de relajaciones extrapoladas de combinaciones convexas de proyecciones por subgradientes sobre familias variables de conjuntos.

Observamos además que, si bien es cierto que los métodos secuenciales clásicos (como el ART) no están bien adaptados para ser paralelizados, vimos también que se han propuesto diferentes técnicas que explotan las bondades del procesamiento en paralelo.

El EMOPSP demostró ser superior al POCS (y a otros métodos conocidos) en cuatro aspectos esenciales [9]:

1. Converge muy eficientemente.

2. No requiere del cálculo de proyecciones exactas.

3. Puede ser implantado en procesadores concurrentes de una manera muy flexible.

4. Puede resolver problemas con un número infinito de restricciones.

Algunas de las técnicas aquí estudiadas han sido aplicadas en otros contextos. Por ejemplo, García-Palomares en [19], aplica estas estrategias a los parámetros de relajación. En otro trabajo del mismo autor, [20], se aplican estas técnicas al estudio del problema de encontrar un punto que satisfaga un sistema lineal de $m$ ecuaciones y $n$ incógnitas, usando métodos de proyección precondicionados y procesamiento en paralelo. Aplicaciones recientes son consideradas en [27] y [41].

\footnotetext{
${ }^{6}$ El EMOPSP verifica la propiedad de monotonicidad de Fejér $\quad(\forall n \in \mathbb{N}) \quad(\forall s \in S)\left\|a_{n+1}-s\right\| \leq \|$ $a_{n}-s \|$, que es una condición fundamental para probar la convergencia de los métodos propuestos para resolver $(2)[5]$.
} 
Los experimentos numéricos llevados a cabo tanto en [9] como en [3] corraboraron la superioridad computacional del método EMOPSP sobre otras versiones del MAP (tanto secuenciales como en paralelo), cuando los mismos se aplican al problema de restauración de imágenes.

\section{Referencias}

[1] Andersen, A.H. (1989) "Algebraic reconstruction in CT from limited views", IEEE Trans. Medical Imaging 8: 50-55.

[2] Andrews, H.C.; Hunt, B.R. (1977) Digital Image Restoration. Prentice-Hall, Englewood Cliffs, NJ.

[3] Arjona, J. (1998) Algoritmos Numéricos para el Problema de Restauración de Imágenes a partir de Métodos de Proyecciones Ortogonales Alternantes. Tesis, Computer Department, Universidad Central de Venezuela, Caracas.

[4] Aubin, J.P. (1993) An Introduction to Nonlinear Analysis. Springer-Verlag, New York.

[5] Bauschke, H.H.; Borwein, J.M. (1996) "On projection algorithms for solving convex feasibility problems", SIAM Rev. 38: 367-426.

[6] Bazaraa, M.S.; Sherali, H.D.; Shetty, C.M. (1993) Nonlinear Programmig, Theory and Algorithms, 2nd. Edition. John Wiley, New York.

[7] Censor, Y. (1988) "Parallel application of block-iterative methods in medical imaging and radiation therapy", Math. Prog. 42(2): 307-325.

[8] Censor, Y.; Zenios,S.A. (1997) Parallel Optimization. Theory, Algorithms, and Applications. Oxford University Press, New York.

[9] Combettes, P.L. (1997) "Convex Set Theoretic Image Recovery by Extrapolated Iterations of Parallel Subgradient Projections", IEEE Trans. Image Processing 6: 493-506.

[10] Combettes, P.L. (1997) "Hilbertian Convex Feasibility Problem: Convergence of Projection Methods", Appl. Math. Optim 35: 311-330.

[11] Combettes, P.L. (1996) "The convex feasibility problem in image recovery", Advances in Imaging and Electron Physics, Academic Press, Editor: P. Hawkes, 95: 155-270, New York.

[12] Combettes, P.L. (1993) "The foundations of set theoric estimation", Proc. IEEE 81:182-208.

[13] Combettes, P.L. (1994) "Inconsistent signal feasibility problems: Least-squares solutions in a product space", IEEE Trans. Signal Processing 42(11): 2955-2966.

[14] P. L. Combettes, P.L.; Puh, H. (1994) "Iterations of Parallel convex projections in Hilbert spaces", Numer. Funct. Anal. Optim. 15: 225-243. 
[15] Deutsch, F. (1992) "The method of alternating orthogonal projections", in S.P. Singh (Ed.) Approximation Theory, Spline Functions and Applications, Kluwer Academic Publishers, Dordrecht: 105-121.

[16] Escalante, R.; Raydan, M. (2000) Alternating Projection Methods: Theory and Applications, Book in preparation, CCCT.

[17] R. Escalante, R.; Raydan, M. (1996) "Dykstra algorithm for a Constrained Leastsquares Matrix Problem", Num. Linear Algebra Appl. 3: 459-471.

[18] Escalante, R.; Raydan, M. (1998) "Dykstra algorithm for a Constrained Least-squares Rectangular Matrix Problem", Computers Math. Applic. 6: 73-79.

[19] García-Palomares, U.M. (1999) "Relaxation in Projection Methods", Preprint, Departamento de Procesos y Sistemas, Universidad Simón Bolívar, Caracas.

[20] García-Palomares, U.M. (1999) "Preconditioning projection methods for solving algebraic linear systems", Numerical Algorithms 21: 157-164.

[21] Gilbert, P. (1972) "Iterative methods for the three-dimensional reconstruction of an object from projections", J. Theoret. Biol. 36(1): 105-117.

[22] González, R.C.; Woods, R.E. (1997) Tratamiento Digital de Imagenes. Adison-Wesley, New York.

[23] Gordon, R.; Bender, R.; Herman, G.T. (1970) "Algebraic reconstruction techniques (ART) for three-dimensional electron microscopy and X-ray photography", J. Theoretical Biol. 29: 471-481.

[24] Gubin, L.G.; Polyak, B.T.; Raik, E.V. (1967) "The method of projections for finding the common point of convex sets", USSR Comp. Math. Phys. 7: 1-24.

[25] Halperin, I. (1962) "The product of projection operators", Acta Sci. Math. (Szeged) 23: $96-99$.

[26] Herman, G.T. (1980) Image Reconstruction from Projections, the Fundamental of Computerized Tomography. Academic Press, New York.

[27] Hocker, T.; Aranovich, G.L.; Donohue, M.D. (2001) "Adsorption-Energy Distribution of Heterogeneous Surfaces Predicted from Projections onto Convex Sets", J. Colloid Interface Sci. 238: 167-176.

[28] Iusem, A.; De Pierro, A. (1986) "Convergence results for an accelerated nonlinear Cimmino algorithm", Numer. Math. 49(4): 367-378.

[29] Katsaggelos, A.K. (1991) Digital Image Restoration. Springer-Verlag, New York.

[30] Luenberger, D. G. (1969) Optimization by Vector Space Methods. John Wiley, New York. 
[31] Mandel, J. (1984) "Convergence of the cyclical relaxation method for linear inequalities", Math. Programming 30: 218-228.

[32] Moreau,J.J. (1963) "Fonctionnelles sous-différentiables", C. R. Acad. Sci. Paris Sér. A 257(26): 4117-4119.

[33] Ottavy, N. (1988) "Strong convergence of projection-like methods in Hilbert spaces", J. Optim. Theory Appl. 56(3): 433-461.

[34] Pierra, G. (1984) "Decomposition through formalization in a product space", Math. Programing 28(1): 96-115.

[35] Rockafellar, R.T. (1970) Convex Analysis. Princeton University Press, Princeton, NJ.

[36] Sezan, M.I.; Stark, H. (1982) "Image restoration by the method of convex projections: Part 2-Applications and Numerical Results", IEEE Trans. Medical Imaging M1-1: 95-101.

[37] Simard, P.Y.; Mailloux, G.E. (1988) "A projection operator for the restoration of divergence-free vector fields", IEEE Trans. Patt. Anal. Machine Intell. 10(2): 248256 .

[38] Stark, H. (1987) Image Recovery: Theory and Application. Academic Press, San Diego, CA.

[39] Trussell, H.J.; Civanlar, M.R. (1984) "The feasible solution in signal restoration", IEEE Trans. Acoust., Speech, Signal Processing 32(2): 201-212.

[40] Von Neumann, J. (1950) The Geometry of Orthogonal Spaces, Vol II. Princeton University Press, Princenton, New Jersey.

[41] Yang, Y.; H. Stark, H. (2001) "Design of Self-Healing Arrays Using Vector-Space Projections", IEEE Trans. on Antennas and Propagation 49(4): 526-534.

[42] Youla, D.C. (1978) "Generalized image restoration by the method of alternating orthogonal projections", IEEE Trans. Circuits Syst., CAS-25: 694-702.

[43] Youla, D.C.; Webb, H. (1982) "Image restoration by the method of convex projections: Part 1-Theory", IEEE Trans. Medical Imaging M1-1: 81-94. 